\title{
Short-term result of arthroscopic synovial excision for dorsal wrist pain in hyperextension associated with synovial hypertrophy
}

Kowshik $\underline{\text { Jain }}^{1}$, FRCS, Raminder $\underline{\text { Singh }}^{1}$, FRCS

\begin{abstract}
INTRODUCTION Arthroscopic excision of the hypertrophic dorsal synovium is performed on patients with dorsal wrist pain in hyperextension. Although dorsal wrist impingement has been described in cadaveric studies, to our knowledge, there is no published clinical data on its treatment with arthroscopic synovial excision. Herein, we present the results of arthroscopic management of this condition in our hospital.

METHOD A total of 13 patients underwent arthroscopic excision of the hypertrophic dorsal impinging synovium. All patients presented with the cardinal symptom of dorsal-radial wrist pain in extreme extension. The diagnoses were made after excluding other causes and confirmed on wrist arthroscopy. Arthroscopy was offered after nonoperative measures failed. The mean postoperative follow-up period was 14 (range 6-31) months.

RESULTS Mean pre- and postoperative quick Disabilities of the Arm, Shoulder and Hand scores were 49 (range 34-82) and 17 (range 0-48), respectively; paired $t$-test revealed a significant difference between the two $(p<0.001)$. Mean postoperative flexion-extension arc and radial-ulnar deviation arc were $120^{\circ}$ and $46^{\circ}$, respectively. Postoperatively, one patient developed complex regional pain syndrome, with tethering of the dorsal branch of the ulnar nerve, which required surgical release, while another patient required revision arthroscopic excision of the impinging tissue. Both patients had good postoperative outcomes. CONCLUSION When treating patients with dorsal wrist pain, dorsal wrist impingement caused by synovial hypertrophy should be included in the differential diagnosis. Arthroscopic excision of the impinging synovium can achieve reliable pain relief with significant functional improvement in the short term, although further research on its long-term benefits is required.
\end{abstract}

Keywords: arthroscopic, impingement, pain, synovium, wrist

\section{INTRODUCTION}

Dorsal wrist impingement is a reproducible clinical presentation of well-localised, dorsal-radial wrist pain. Patients with dorsal wrist impingement generally report pain in a precise location in the dorsal-radial location of the wrist; this pain is experienced particularly in loaded extension, such as when pushing up from a chair. ${ }^{(1)}$ Diagnosis is established via thorough history-taking and physical examination. Investigations in the form of radiography or magnetic resonance imaging are only helpful in some cases. Although dorsal wrist impingement has been described in cadaveric studies, ${ }^{(1)}$ to the best of our knowledge, there is currently no published clinical data on its treatment with arthroscopic synovial excision. Herein, we present the results of arthroscopic synovial excision performed in patients who presented to our hospital with dorsal wrist pain in extension.

\section{METHODS}

Between 2008 and 2010, 13 consecutive patients with dorsal wrist impingement were treated in our hospital. All diagnoses were made after obtaining each patient's typical history of dorsal wrist pain in hyperextension. Clinical assessment of the patients included history-taking and clinical examination. The mean age of the patients was 42 (range 22-60) years. The male to female ratio was 9:4 and the ratio of occurrence of right-sided to left-sided wrist impingement was 10:3. Of the 13 patients, 10 presented with symptoms on their dominant side. The mean duration of symptom was 9 (range 1.5-24.0) months. There was no history of injury in 4 of the 13 patients, while the remaining 9 patients recalled some form of injury - falling on an outstretched hand $(n=4)$, falling on a dorsiflexed wrist $(n=1)$, wrist striking a cupboard $(n=1)$, and pain noticed while lifting a bag of sand $(n=1)$, after punching a bag $(\mathrm{n}=1)$ and while hammering $(\mathrm{n}=1)$. All patients presented with the cardinal symptom of dorsal-radial wrist pain in hyperextension.

The clinical findings were nonspecific, with five patients having positive Kirk-Watson tests. Of these five patients, only one had a partial tear of the scapholunate ligament (SLL) on arthroscopy; in the other four patients, the SLL was intact. SLL was also intact in the eight patients who did not have a positive Kirk-Watson test. Both the triangular fibrocartilage complex (TFCC) and lunotriquetral ligament (LTL) were intact in all 13 patients. Wrist radiography was unremarkable in all, except for one patient in whom a healed distal radial fracture was identified. Magnetic resonance arthrography performed in two patients revealed a TFCC tear in one patient and minor degenerative changes in the other.

All patients received a trial of conservative management in the form of splinting, steroid injection and/or physiotherapy. The mean duration of conservative management was 12 (range 1.5-24.0) months. Three patients received one steroid

${ }^{1}$ Wrexham Maelor Hospital, Wrexham, United Kingdom

Correspondence: Dr Kowshik Jain, 6 Bron-y-nant, Wrexham, United Kingdom, LL13 7TX. kowshikjain@gmail.com 


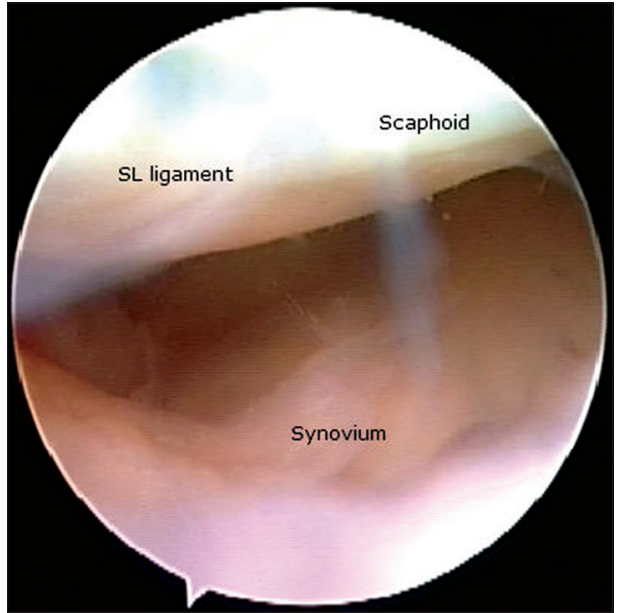

Fig. 1 Endoscopic image shows a hypertrophic synovium, seen dorsally from the 3-4 portal. SL: scapholunate

injection each and physiotherapy, three received splinting and physiotherapy, while the rest received only physiotherapy. All 13 patients did not derive any significant benefit from conservative management, including the use of steroid injections.

Standard 3, 4 and 6R arthroscopic portals were used in wrist arthroscopy. Midcarpal arthroscopy was performed as a day case in all 13 patients. The pale, hypertrophied and thickened dorsal impinging synovium was identified and excised to the level of the wrist joint capsule using either mechanical synovial shavers or a radiofrequency wand (Figs. 1 \& 2). Postoperatively, all the patients were referred to hand therapists for early range of motion and strengthening exercises.

\section{RESULTS}

Postoperative evaluation included assessments of the range of movement, functionality (in the form of quick Disabilities of the Arm, Shoulder and Hand [DASH] scores) and level of dorsal wrist pain in hyperextension (Table I). The mean duration of postoperative follow-up was 14 (range 6-31) months. Improvement in dorsal wrist pain following the operation was observed in 12 patients. Data on postoperative pain was not available for one patient. At postoperative assessment, seven patients experienced no pain, two patients had mild wrist pain and three had moderate wrist pain.

Data on postoperative range of movement was available for 11 of the 13 patients. The mean postoperative flexion was $56^{\circ}$ (range $40^{\circ}-80^{\circ}$ ), while the mean extension was $64^{\circ}$ (range $\left.50^{\circ}-70^{\circ}\right)$. The mean flexion-extension arc was $120^{\circ}$, which is comparable to the flexion-extension range in healthy patients..$^{(2,3)}$ The mean ulnar deviation was $25^{\circ}$ (range $6^{\circ}-40^{\circ}$ ) and the mean radial deviation was $21^{\circ}\left(\right.$ range of $\left.5^{\circ}-31^{\circ}\right)$. Pre- and postoperative quick DASH scores were available for 12 of the 13 patients. The mean pre- and postoperative quick DASH scores were $49 \pm 14$ (range 34-82) and $17 \pm 17$ (range 0-48), respectively. Paired $t$-test revealed a significant difference between the pre- and postoperative quick DASH scores $(\mathrm{p}<0.0001)$.

Two patients developed postoperative complications. One patient who experienced persistent symptoms required revision

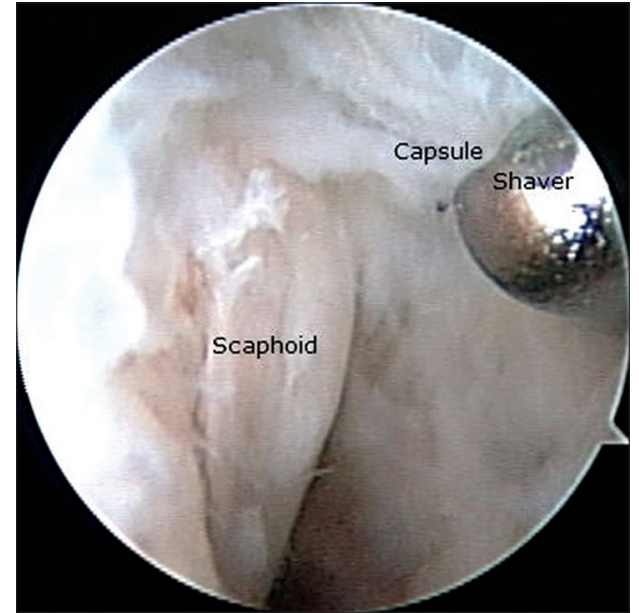

Fig. 2 Endoscopic image shows the wrist joint capsule after synovial excision.

Table I. Pre- and postoperative quick Disabilities of the Arm, Shoulder and Hand (DASH) scores, level of postoperative pain and postoperative range of movement.

\begin{tabular}{|c|c|c|c|c|c|}
\hline \multirow[t]{2}{*}{ Patient } & \multicolumn{2}{|c|}{ Quick DASH score } & \multirow{2}{*}{$\begin{array}{l}\text { Postop } \\
\text { pain }\end{array}$} & \multirow{2}{*}{$\begin{array}{l}\text { Postop } \\
\text { VF/DF }\end{array}$} & \multirow{2}{*}{$\begin{array}{l}\text { Postop } \\
\text { UD/RD }\end{array}$} \\
\hline & Preop & Postop & & & \\
\hline 1 & 59.1 & 38.6 & Moderate & $50 / 70$ & $20 / 20$ \\
\hline 2 & 45.5 & 20.5 & Mild & $72 / 69$ & $25 / 20$ \\
\hline 3 & 36.4 & 0 & None & $40 / 50$ & $28 / 24$ \\
\hline 4 & 61.4 & 34.1 & Moderate & $42 / 70$ & 06/05 \\
\hline 5 & 45.5 & 20.3 & None & $70 / 60$ & $30 / 30$ \\
\hline 6 & 36.4 & 4.5 & None & $45 / 50$ & $40 / 20$ \\
\hline 7 & 45.5 & 22.7 & Mild & NA & NA \\
\hline 8 & 54.5 & 47.7 & Moderate & $55 / 73$ & $27 / 19$ \\
\hline 9 & 40.9 & 11.4 & None & NA & NA \\
\hline 10 & 34.1 & 2.3 & None & $50 / 68$ & $25 / 31$ \\
\hline 11 & NA & NA & NA & $60 / 60$ & $25 / 21$ \\
\hline 12 & 47.7 & 0 & None & $56 / 64$ & $25 / 21$ \\
\hline 13 & 82.5 & 0 & None & $80 / 70$ & $20 / 20$ \\
\hline
\end{tabular}

DF: dorsiflexion; NA: not available; RD: radial deviation; UD: ulnar deviation; VF: volar flexion

wrist arthroscopy and removal of the synovium. The patient showed marked improvement after the second operation. The other patient developed complex regional pain syndrome, with tethering of the superficial branch of the ulnar nerve, requiring surgical release. The patient's symptoms improved following the second operation.

\section{DISCUSSION}

Chronic wrist pain with no detectable abnormalities on radiography is common. ${ }^{(4)}$ As the evaluation of chronic wrist pain is challenging, thorough history-taking and physical examination are essential. ${ }^{(5)}$ Although some patients respond to conservative treatment, a subset continues to have chronic pain even with conservative treatment. For this subset of patients, wrist arthroscopy may be useful for determining the cause of the occult wrist pain and for treating the pain.

Chronic dorsal wrist pain in hyperextension is called dorsal wrist syndrome. ${ }^{(6)}$ The proposed aetiologies for this are rotary 
subluxation of the scaphoid, ${ }^{(7)}$ synovitis, ${ }^{(4)}$ extensor retinaculum impingement ${ }^{(8)}$ and dorsal wrist capsule impingement. ${ }^{(1)}$ The treatment for dorsal wrist syndrome varies according to its aetiology. In 2004, Yasuda et al described 20 cases in which open excision of the dorsal wrist capsules, with excision of the posterior interosseous nerve, was performed; postoperatively, $60 \%$ of their patients reported an absence of pain. ${ }^{(9)}$ In a 2008 cadaveric study, Henry proposed that arthroscopic excision of the dorsal wrist capsule was a reliable solution for the treatment of dorsal wrist impingement. ${ }^{(1)}$ In the same year, Wollstein et al published the results of dorsal capsulodesis for radial wrist pain in 88 patients; $80 \%$ of their patients described improvement in pain and function, while $25 \%$ required further operation. ${ }^{(4)}$ VanHeest et al, who described partial distal resection of the extensor retinaculum in five athletes with dorsal wrist pain in hyperextension, reported complete pain relief and full return to sports in all five athletes. ${ }^{\left({ }^{8}\right)}$ In 2012, Tan et al found that arthroscopic debridement alone could achieve improvement in wrist pain in patients with intercarpal and TFCC tears. ${ }^{(10)}$ Similarly, Weiss et al reported high rates of short-term symptomatic relief in both complete and incomplete SLL tears using arthroscopic debridement alone. ${ }^{(11)}$

To the best of our knowledge, this is the first report on the use of arthroscopic synovial excision for the treatment of chronic dorsal wrist pain in hyperextension. Postoperative quick DASH scores were significantly higher than the preoperative scores among the 12 patients whose scores were available. In the present study, radiofrequency wands at a very low intensity were used in earlier cases, and resulted in early postoperative pain and possibly, complex regional pain syndrome. In later cases, excision was done exclusively with mechanical synovial shavers, resulting in quicker postoperative pain relief. Thus, we recommend the use of mechanical synovial shavers for the excision of the synovium.
The limitations of the present study are the small number of patients, the use of a non-wrist-specific outcome measure, the short follow-up period, and the nonstandardised preoperative treatment and postoperative follow-up plans. Despite these limitations, the study still adds valuable knowledge regarding the infrequent problem of chronic dorsal wrist pain in hyperextension.

To conclude, arthroscopic excision of hypertrophic dorsal synovium using mechanical synovial shavers provides short-term reliable improvement in function and short-term pain relief in patients with chronic dorsal wrist pain in hyperextension. Further research on the long-term benefits of this treatment is required.

\section{REFERENCES}

1. Henry M. Arthroscopic management of dorsal wrist impingement. J Hand Surg Am 2008; 33:1201-4.

2. Ruby LK, Cooney WP 3rd, An KN, Linscheid RL, Chao EY. Relative motion of selected carpal bones: a kinematic analysis of the normal wrist. J Hand Surg Am 1988; 13:1-10.

3. Youm Y, McMurtry RY, Flatt AE, Gillepsie TE. Kinematics of the wrist. I. An experimental study of radial-ulnar deviation and flexion-extension. J Bone Joint Surg Am 1978; 60:423-31.

4. Wollstein R, Watson HK, Wear-Maggitti K, Schmidt S, Carlson L. Surgical technique for the treatment of radial wrist pain. Scand J Plast Reconstr Surg Hand Surg 2008; 42:149-52.

5. Nagle DJ. Evaluation of chronic wrist pain. J Am Acad Orthop Surg 2000; 8:45-55.

6. Weinzweig J, Watson HK. Dorsal wrist syndrome repair. In: Watson HK, Weinzweig J. The Wrist. Philadelphia: Lippincott Williams \& Wilkins, 2001: 829-31.

7. Watson H, Ottoni L, Pitts EC, Handal AG. Rotary subluxation of the scaphoid: a spectrum of instability. J Hand Surg Br 1993; 18:62-4.

8. VanHeest AE, Luger NM, House JH, Vener M. Extensor retinaculum impingement in the athlete: a new diagnosis. Am J Sports Med 2007; 35:2126-30

9. Yasuda M, Masada K, Takeuchi E. Dorsal wrist syndrome repair. Hand Surg 2004; 9:45-8

10. Tan SW, Ng SW, Tan SH, Teoh LC. Arthroscopic debridement of intercarpal ligament and triangular fibrocartilage complex tears. Singapore Med J 2012; 53:188-91.

11. Weiss AP, Sachar K, Glowacki KA. Arthroscopic debridement alone for intercarpal ligament tears. J Hand Surg Am 1997; 22:344-9. 